PRE-PRINT: Bukovac, Z, Dorin, A., Dyer A.G., "A-Bees See: A Simulation to Assess Social Bee Visual Attention During Complex Search Tasks", in Proceedings of the 12th European Conference on Artificial Life (ECAL 2013), Liò et al (eds.) Taormina, Italy, 2-6 Sept., MIT Press, 8 pp. (To appear, accepted June 2013)

\title{
A-Bees See: A Simulation to Assess Social Bee Visual Attention During Complex Search Tasks
}

\author{
Zoe Bukovac $^{1}$, Alan Dorin ${ }^{1}$ and Adrian G. Dyer ${ }^{1,2}$ \\ ${ }^{1}$ Monash University, Clayton, Australia 3800 \\ ${ }^{2}$ RMIT University, Melbourne, Australia 3000 \\ zoe.bukovac / alan.dorin@monash.edu (corresponding author), adrian.dyer@rmit.edu.au
}

\begin{abstract}
Foraging bees often search in complex natural environments for "target" flowers that they have learnt provide nectar rewards. To maximise efficiency, bees must avoid landing on "distractor" flowers that do not offer rewards, as this potentially wastes time and energy. This paper reports on artificial-life inspired agent-based simulations of two contrasting approaches different bee species use to scan for targets in a scene containing many flowers. The two scanning approaches simulated are a parallel scan typical of bumblebees that is not slowed by distractors, and a serial scan typical of honeybees that is faster than parallel scan for single element processing, but is slowed by the presence of distractor flowers. The simulations were conducted over a range of target densities, and over a range of target/distractor ratios, to evaluate the types of environment in which each scan mechanism is most effective. Serial scan was found to be generally more effective in environments populated with a single type of rewarding species of flower, and parallel scan appears to be relatively more effective in environments populated with a mix of rewarding and unrewarding flowers. Our results support the hypothesis that environmental factors led to the evolution of different visual processing mechanisms in honeybees and bumblebees. This establishes a firm basis for psychophysical research exploring how and why the two different processing mechanisms may have evolved in these animals.
\end{abstract}

\section{Introduction and Previous Work}

Foraging worker bees collect nutrition to sustain a beehive. Individual bees travel on "bouts" between their hive and flowers that may present nectar and pollen as nutritional rewards. Some bee species like honeybees (Apis mellifera) have colonies of foragers numbering in the thousands; while other species like bumblebees (Bombus terrestris) typically have less than 100 foragers (Frisch 1967, p.7; Duchateau and Velthuis 1988). Differences in search strategy between individual bees are amplified many times by the numerous bouts an individual travels during a day, many more times depending on the number of bees in a colony sharing that strategy, and more times still over a season or the life of a colony. The evolutionary relationship of different bee species and flowers is likely to have endured over many millions of years (Dyer, Boyd-Gerny et al. 2012), suggesting that selective pressure to evolve optimal solutions may exist in current day populations (e.g. examples throughout (Lythgoe
1979)). The search strategy employed should therefore be adapted to local environmental conditions, maximising the net flow of energy into the hive to enable survival and reproductive success of the queen bee. Costs associated with unnecessary workers, or excessive flight and flower handling should be avoided, but this is not straightforward in complex natural environments (Burns and Dyer 2008).

During a foraging bout, bees search for target flowers that they have learnt provide nectar rewards. Many social bees, like honeybees and bumblebees, exhibit flower constancy and tend to forage consistently from one type of rewarding flower as long as it continues to present rewards (Chittka, Thomson et al. 1999). However, in complex environments with many flowers, bees must avoid landing on unrewarding distractor flowers as this wastes time and energy (Burns and Dyer 2008). When the colours of target and distractor flowers are very different (e.g. blue and yellow as seen by humans), and there are only two flowers to choose between, bees can accurately assess the type of a flower presented to them (Giurfa 2004; Dyer, Spaethe et al. 2008). However, it isn't currently well understood how bees make decisions in complex environments containing many flowers of different types, colours, target/distractor ratios and arrangements. For instance, in tropical forests, single trees with thousands of simultaneously blooming rewarding flowers, potential targets, may appear (Fig. 1a), and distractor flowers are not intermingled among them (Clark 1994). However, in temperate environments, diverse carpets of small herbaceous plants may include a scattered few targets peppered among numerous distractor species (Fig. 1b), or a uniform carpet of a single species may occur that is many meters across depending upon location or season (Fig. 1c).

Apart from the obvious biological interest foraging raises (Pyke 1984), understanding bee foraging has many practical implications for agriculture where globally, crop beepollination is directly responsible for $35 \%$ of worldwide food production and is worth an estimated 153 billion Euro annually (Kjøhl, Nielsen et al. 2011, pp.1-49). Bee pollination is also essential for natural ecosystem management (Hegland, Nielsen et al. 2009). It is therefore imperative that we understand how different bee species operate in different environments. This also provides insight into how potential 
temporal or spatial mismatches in bee-pollinator and/or flower blooming may be affected by predicted changes in environmental conditions (ibid.). Furthermore, understanding bee visual attention mechanisms is relevant to artificial vision and search system design (Srinivasan 2011).

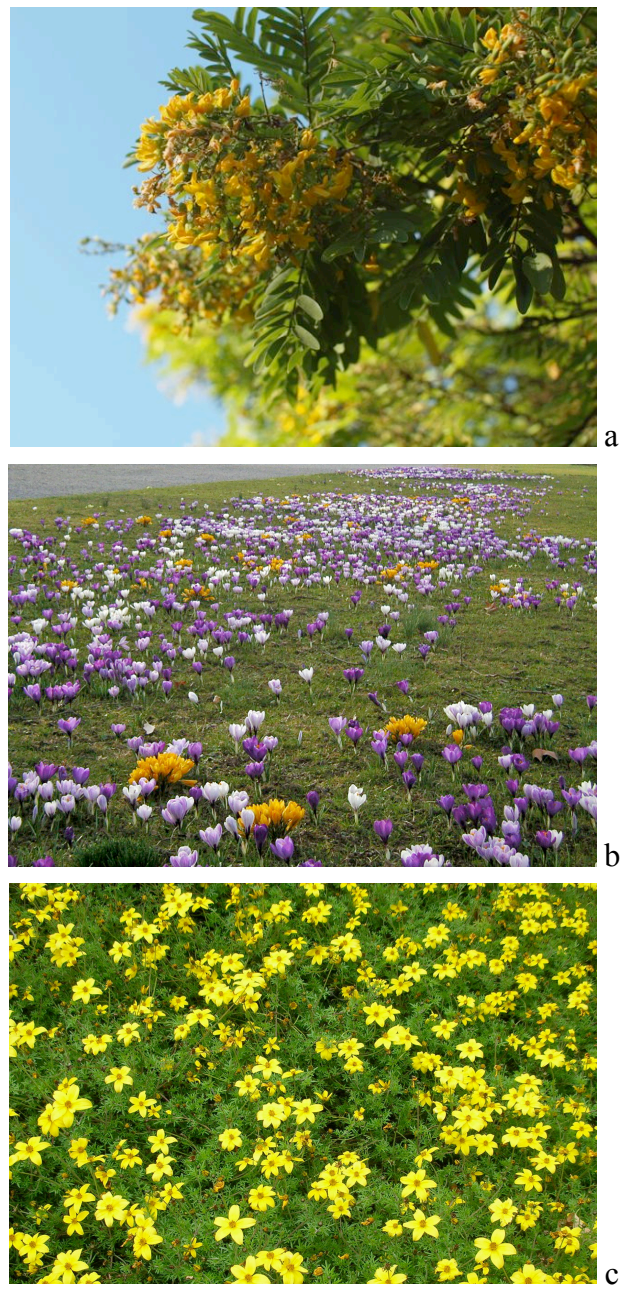

Figure 1: Sample flower distributions: (a) flowering tree, (b) patchy temperate carpet, (c) uniform temperate carpet.

Biological background. Following recent experiments with live free-flying bees, Morawetz and Spaethe (2012) propose that bumblebees (Bombus terrestris) conduct what appears to be a limited parallel style visual scan for targets that is not significantly slowed by increasing the number of distractors, while honeybees (Apis mellifera) use a serial styled scan that is faster for decisions where only targets are present, but is slowed considerably when distractors that must also be processed by the visual system are present. These different spatial attention mechanisms are potentially required to allow an organism with finite information processing capacity to handle the essentially infinite complexity of applying vision in natural environments (Treisman and Gelade 1980; Treisman and Gormican 1988; VanRullen, Carlson et al. 2007).

The study on free-flying honeybees and bumblebees (Morawetz and Spaethe 2012) used differently coloured paper targets known to stimulate the trichromatic (UV, blue and green sensitive (Dyer, Paulk et al. 2011)) colour processing system of bees. This type of behavioral testing relies on the fact that individual bees can be trained to visit a target colour by associating a sucrose solution which the bees collect as nutrition, whilst the distractor stimuli offer no reward to bees who therefore avoid this colour. This scenario is biologically relevant since flowers may only offer rewards in certain temporal cycles, and there are some that mimic rewarding flowers and try to obtain pollination through deception (Dafni 1984; Dyer, Paulk et al. 2011). The Morawetz and Spaethe (2012) study was conducted in a controlled arena with stimuli presented at set visual angles. The number of targets and distractors was systematically varied. The results showed that increasing the number of distractors led to a significant increase in the decision-making time for honeybees, consistent with theories of a serial search mechanism (Treisman and Gelade 1980; Treisman and Gormican 1988; VanRullen, Carlson et al. 2007). However, in bumblebees a different processing system was observed. While decisions for finding a target with only a single distractor were about 1.5 times as long as for honeybees, increasing the number of distractors did not significantly affect the decision-making time of bumblebees. This type of decision-making is consistent with a parallel visual search (ibid.). In the current study we use simulations to test the implications on nectar gathering effectiveness, as a biologically relevant measure of fitness, for parallel and serial scanning mechanisms.

Simulation background. Experiments with free-flying bees require marking and tracking individual animals moving freely in 3D space, making it difficult to collect sufficient reliable data to answer iterative questions about optimal mechanisms in multiple environments. Hence, we employ an agent-based model (ABM, or individual-based model, IBM) simulating parallel and serial scanning bees in different environments. Our artificial bees (a-bees) search a grid world populated by target and distractor flowers. We systematically sweep through a biologically relevant range of target densities and target/distractor relative abundances, aiming to determine the environmental floral distributions in which each visual scan mechanism is likely to be effective in real world scenarios. The simulations allow us to interpret the factors that influence how and why bees make decisions, and the subsequent colony-level benefits that may act as biologically relevant factors for reproductive success (Burns 2005; Burns and Dyer 2008).

Where bee behaviour varies between individuals or where local environmental conditions influence individual decisionmaking, ABMs offer a powerful approach for understanding the intricate interactions and emergent outcomes of these complex systems (Huston, DeAngelis et al. 1988; Judson 1994; Grimm 1999; DeAngelis and Mooij 2005; Grimm and Railsback 2005; Grimm, Revilla et al. 2005; Dorin, Korb et al. 2008). ABMs have been used to model bee behaviour since the 1980s (Hogeweg and Hesper 1983). For example, they have been used to understand bee foraging strategies in keeping with empirical data whilst considering recruitment, 
homing and memory of food source location (Vries and Biesmeijer 1998); and to show that the benefits of recruitment by honeybees is dependent on the density of flowers within certain environments (Dornhaus, Klügl et al. 2006). ABMs have also been applied to understand the impact of flower constancy under conditions where flower rewards become available or unavailable cyclically (Dyer, Dorin et al. 2012).

In this current study we use ABMs to understand the potential colony level benefits of the empirically determined visual scanning behaviour discovered in honeybees and bumblebees. Specifically, we hypothesise that if environmental conditions like flower density have been a factor in the evolution of different bee species' visual search mechanisms (Dyer, Spaethe et al. 2008; Morawetz and Spaethe 2012), then there should be evidence of biologically plausible flower distributions best suited to foraging by bees employing the different mechanisms.

\section{Simulation Method}

The ABM we have designed simulates the components pertinent to testing the hypothesis just stated, while eliminating irrelevant factors - KISS. ${ }^{1}$ In detailing the simulation here we discuss aspects of real bee behavior included or excluded to test our hypotheses. The system was implemented entirely in the $\mathrm{C}$ programming language but there is nothing in our model that could not be built in any other similar language by following the description below.

\section{Artificial-Bees (A-Bees)}

Bees are central place feeders that leave their hive to search for nutrition. We model bees as software agents, a-bees, foraging within a virtual bounded foraging patch. The patch is uniformly divided into square grid cells. At most one virtual flower can occupy a grid cell. An a-bee also occupies a single grid cell with or without a flower. In this section we detail abee behaviour, the simulated environment and the relationship of these aspects of our model to reality.

We model bee colonies of 60 foragers. The exact number is not critical since we eliminate inter-agent communication and population density effects by ensuring that, in essence, each abee exists in a world of its own. Inter-agent effects are complicating factors that would change the viability of different visual scan mechanisms for hives of different size and under different environmental conditions. In keeping with KISS described above, the simulation eliminates these to reduce one key problem to its basic form.

Honeybees have evolved a complex language for communicating target whereabouts to one another (Frisch 1967, pp.321-328). This is of particular benefit in environments where targets appear in large clusters (Dornhaus, Klügl et al. 2006). This communication system is likely to impact on the effectiveness of different bee species'

\footnotetext{
${ }^{1}$ Keep It Simple, Stupid (Axelrod 2003).
}

visual scanning techniques, but we have avoided introducing it here in order to establish a baseline for comparing only the visual scans of honeybees against those of bumblebees (Dornhaus and Chittka 1999).

Bees can use vision and olfaction to help find flowers; e.g. (Streinzer, Paulus et al. 2009). Our model only considers visual scan. Our a-bees can distinguish between targets and distractors with $100 \%$ accuracy. This is biologically plausible for saliently different colours (Giurfa 2004; Dyer, Paulk et al. 2011).

Bee spatial acuity is relatively poor compared to human vision, and in real life bumblebees can only detect a plant's cluster of 3-5 flowers (each flower of $2.5 \mathrm{~cm}$ diameter) at a distance of approximately $0.7 \mathrm{~m}$ (Dyer, Spaethe et al. 2008; Wertlen, Niggebrugge et al. 2008). Detection appears to approximate a step function, so we model it as distances $>0.7 \mathrm{~m}$ - not detected, distance $<0.7 \mathrm{~m}-100 \%$ chance of detecting a flower that is present. Our foraging patch model is based on a grid world of square cells of dimension $0.35 \mathrm{~m}$, so an a-bee can see flowers in its Moore neighbourhood $(8+1$ cells, $n=1$ ), but no further.

An individual a-bee keeps track of the flowers it has visited and will not visit a flower twice in a single foraging simulation. This is biologically plausible and the ability is an important aspect of bee foraging behavior (Giurfa, Núñez et al. 1994). An a-bee will move into a grid cell towards a target if it has not visited that target before. It will then visit that target (to collect the modeled nutritional reward), taking a parameterised amount of time, VisitTime. Real bees do have a central foveal region in which they may see detail better. However its operation in complex environments for different species has been poorly studied (but see (Morawetz and Spaethe 2012)). For simplicity we eliminated this factor here; a-bees are not directionally biased in their visual scan. ${ }^{2} \mathrm{We}$ model the two bee scan mechanisms as follows.

Parallel scan. An a-bee using a parallel scan (Fig. 2) processes all of the information it sees about flower locations in its visible range simultaneously. This process takes the "parallel a-bee" a constant amount of time, ParallelTime, regardless of how many flowers it can see and regardless of whether these are distractors or targets. It is as if in ParallelTime the bee forms a mental image of the whole visible scene and recognises the closest target, while ignoring all non-targets.

A parallel a-bee will move towards an unvisited target it sees that is drawn from a uniform random distribution of available target flowers. If it finds no target, or no flowers at all, it will conduct a random walk as discussed shortly.

2 However, we did test the impact of bias in the a-bees' preferred direction of travel (below). 


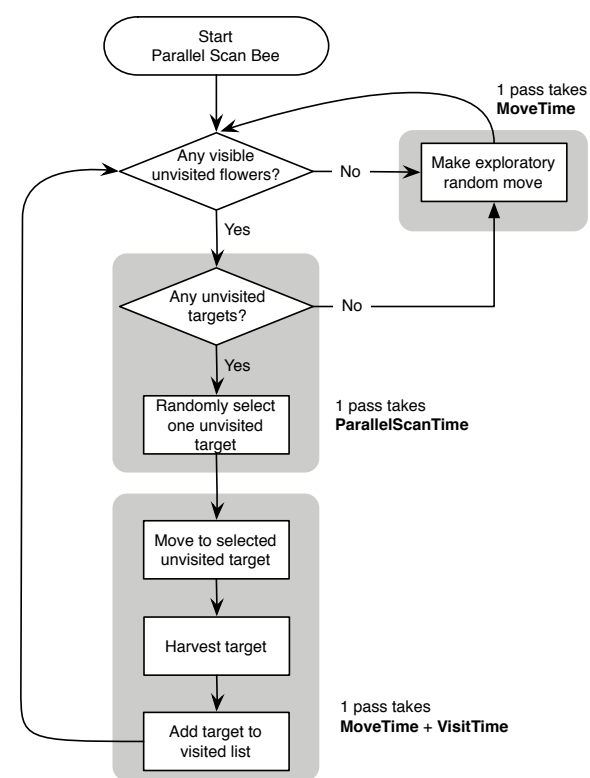

Figure 2: The foraging cycle of a parallel visual scan a-bee.

Serial scan. An a-bee using a serial scan (Fig. 3) examines one flower in its visible region at a time, selecting flowers to investigate from a uniform random distribution across available flowers. It takes this "serial a-bee" the parameter value SerialTime to examine each flower, until it finds a target, or until it has examined all the flowers it can see and finds no targets. A serial a-bee always moves towards the first unvisited target it sees in its scan. It stops scanning as soon as it finds an unvisited target. In the absence of unvisited targets or any flowers at all, the serial a-bee conducts a random walk as discussed below.

A-bee movement. As long as all a-bees, regardless of parallel/serial visual scanning mechanism, apply the same movement strategies, we can assess the relative benefits of the scanning mechanisms free from interference introduced by movement strategies that are themselves complex and worth independent study (Waddington 1980). Hence it is especially important here to eliminate unnecessary complications from the simulation. Since we are concerned with bee decisionmaking times and unconcerned about bee travel times, we don't require a-bees to return to a hive and therefore don't model one. A-bees do not run out of nectar storage; they accrue it indefinitely during a simulation run.

In parameter MoveTime simulation time-steps an a-bee can move into any cell in its Moore neighbourhood, or choose to remain in its current cell. But in which direction should it head? If it sees a target flower, as discussed above, it will move towards that. In the absence of target flowers, a-bees conduct either a random walk or a biased random walk around their Moore neighbourhood depending on the experiment.

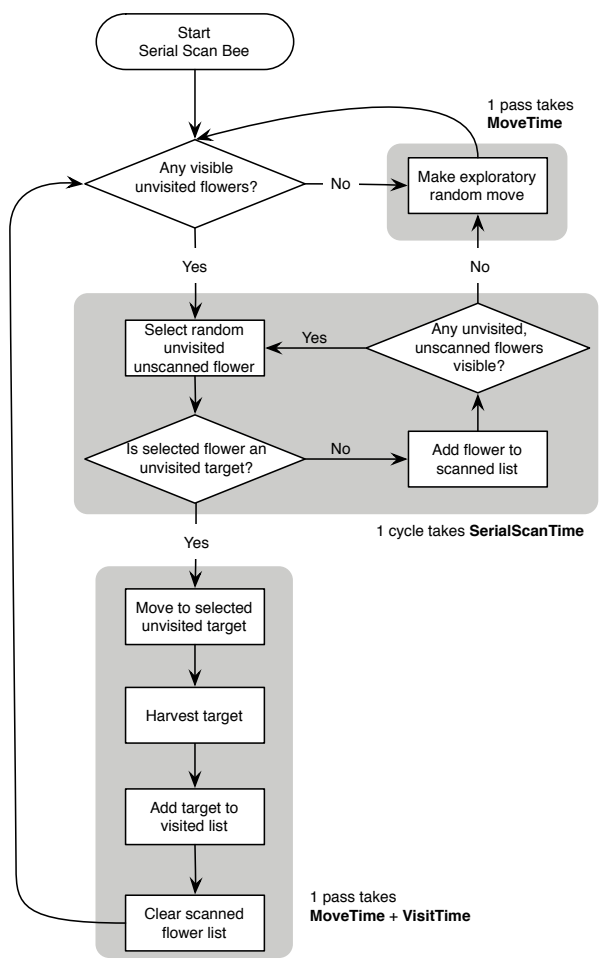

Figure 3: The foraging cycle of a serial visual scan a-bee.

While an ordinary random walk between grid cells is not a biologically realistic foraging strategy, it provides a convenient baseline against which we compare the impact of a biased random walk derived from a study of real bees' directional preferences (Waddington 1980, Fig. 1). The probability of a biased random walking a-bee selecting a specific cell from its Moore neighbours in the absence of suitable target flowers is given in Fig. 4. As can be seen, it prefers to continue straight ahead, but is not completely averse to changing direction. We investigated these two navigational strategies to determine if they had any impact on the relative success of the visual scan techniques.

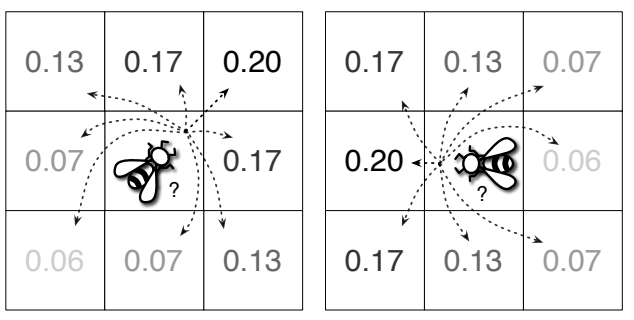

Figure 4: Probability of movement relative to (example) current headings of an a-bee following a biased random walk. Values calculated from (Waddington 1980, Fig. 1).

\section{Artificial Foraging Environments}

A colony's foraging environment is modeled as a bounded world of $571 \times 571$ square grid cells. Cells represent $0.35 \mathrm{~m}^{2}$. Hence, the simulated foraging site is $200 \mathrm{~m}$ across. 
Target flowers offer a reward of 1 unit to every a-bee that visits them. Distractor flowers offer no reward. ${ }^{3}$ Flowers are distributed differently across the patch depending on the experiment, as described in the next section.

\section{Experiments}

We conducted experiments to determine the impact of various flower distributions on the relative success of colonies composed either entirely of parallel a-bees, or entirely of serial a-bees. The simulation parameters described below are summarised in Table 1.

Target-only experiments. In some environments, bees only encounter a single flowering species during a foraging bout (Fig. 1a \& 1c). This is often the case under tropical conditions where massive flowering trees can be encountered, or in homogeneous agricultural fields. In experiments conducted to model these conditions, colonies of serial and parallel a-bees foraged in patches containing only targets. The number of target flowers in the patch was increased systematically to occupy from $0 \%$ to $100 \%$ of the grid cells. Serial and parallel visual scanning mechanisms were compared at each density. Flower positions were distributed uniform randomly across the patch in each case. These experiments were primarily used to inform the choice of target density to be used in the Target and distractor experiments described next.

Target and distractor experiments. In order to ascertain the relative impact of distractor flowers on the serial and parallel visual scanning mechanisms, distractors were placed in the foraging patch among a fixed number of targets. In our simulation, distractor flowers form a single class of non-target flower. In the wild there may be many species of distractor. However, our simulation remains biologically relevant since, as detailed in section Biological background, when distractor flowers are of saliently different colour to targets, bees reliably distinguish between them.

For the Target and distractor experiments, the density of target flowers in our simulated patch was fixed at $10 \%$ of the grid cells. There were three reasons for this density choice:

(i) Since we are measuring the relative success of the two visual scanning mechanisms by recording the total number of reward units gathered during the simulations, we always require some target flowers for a-bees to harvest.

(ii) To maximise our ability to distinguish differences between visual scan mechanisms we need to provide sufficient targets that foraging a-bees do not exhaust the supply of unvisited targets during a simulation run.

(iii) We need to sweep across a wide range of distractor flower densities. By fixing targets at $10 \%$ we have from $0 \%$ up to the remaining $90 \%$ of grid cells to populate with distractors.

3 Actually, a-bees never visit distractors as they distinguish flower types with $100 \%$ accuracy. This is consistent with empirical data for bees visiting saliently different coloured flowers (Giurfa 2004).
Our Target-only experiment results demonstrated that $10 \%$ target density met all three requirements. These results are described below. Hence, during Target and distractor experiments, the number of distractors in the patch was increased systematically to occupy from $0 \%$ to $90 \%$ of the grid cells. The two visual scanning mechanisms were tested at each distractor density. Flowers were distributed uniform randomly across the patch in each case, simulating a temperate environment with different numbers of distractors positioned among targets (Fig. 1b).

\begin{tabular}{ll} 
Environment & \\
\hline Patch size & $571 \times 571$ cells, bounded \\
\hline Patch grid cell size & $0.35 \times 0.35 \mathrm{~m}$ \\
\hline Colony size & 60 parallel, or 60 serial scan a-bees
\end{tabular}

\begin{tabular}{ll} 
A-bees & \\
\hline $\begin{array}{l}\text { Flower presence } \\
\text { detection accuracy }\end{array}$ & $\begin{array}{l}100 \% \text { from neighbouring cell or } \\
\text { cell shared with a flower }\end{array}$ \\
\hline $\begin{array}{l}\text { Flower type } \\
\text { recognition accuracy }\end{array}$ & $\begin{array}{l}100 \% \text { from neighbouring cell or } \\
\text { cell shared with a flower }\end{array}$ \\
\hline $\begin{array}{l}\text { Storage capacity } \\
\text { Infinite }\end{array}$ \\
$\begin{array}{l}\text { Visited flower } \\
\text { memory length }\end{array}$ & $\begin{array}{l}\text { Every flower visited in a } \\
\text { simulation run }\end{array}$ \\
\hline $\begin{array}{l}\text { Flower visit } \\
\text { (VisitTime) }\end{array}$ & 1 simulation time step \\
\hline $\begin{array}{l}\text { Complete field of view } \\
\text { parallel scan } \\
\text { (ParallelTime) }\end{array}$ & 3 simulation time steps \\
\hline $\begin{array}{l}\text { Single serial scan } \\
\text { flower examination } \\
\text { (SerialTime) }\end{array}$ & 2 simulation time steps \\
\hline $\begin{array}{l}\text { Movement in Moore } \\
\text { neighbourhood } \\
\text { (MoveTime) }\end{array}$ & 1 simulation time step \\
\begin{tabular}{l} 
Simulation \\
\hline Duration
\end{tabular} & \\
\hline Number of runs & 20 per data point \\
\hline
\end{tabular}

Table 1: Main simulation parameters.

\section{Simulation verification}

We tested that the simulation behaved according to the specifications above. Tests included that a-bees were:

- Correctly following their respective visual scanning mechanisms in assessing visible flowers;

- Remembering visited target flowers;

- Not exhausting the global target flower supply;

- Not exhausting the local target flower supply.

Where possible we also compared analytically derived values to simulation results. To ensure our a-bees' simplistic random walk navigation strategy did not influence the relative success of the tested scanning mechanisms, we compared the results of this navigation strategy against a more plausible biased walk derived from empirical data. See the Results for a discussion of these experiments. 


\section{Results}

\section{Target only experiments}

In these experiments the foraging patch was filled purely with target flowers. The number of target flowers was increased systematically from $10 \%$ to $100 \%$ of the grid cells in the patch. The effects of this increase on the amount of nectar collected are shown for colonies of parallel and serial a-bees (Fig. 5). All bees in these experiments executed an ordinary random walk.

At target densities of 1 or $2 \%$ there was a relatively low rate of nectar collection by a-bees with either serial or parallel search mechanisms (Fig. 5). A comparison of nectar collection success for colonies of a-bees using parallel (mean 4920 \pm 103 units) or serial (mean $5323 \pm 118$ units) visual search considering $10 \%$ target density and no distractors was conducted with a non-parametric Mann-Whitney U test (SPSS v15.0: IBM, Chicago, USA) for 2-independent groups of $\mathrm{N}=20$ runs/group $(\mathrm{Z}=-5.383, \mathrm{p}<0.001)$. Thus at $10 \%$ target density, a-bees with a serial search mechanism collected significantly more nutrition. As target flower density increases in the presence of no distractors, the serial search becomes increasingly more effective than the parallel search mechanism (Fig. 5).

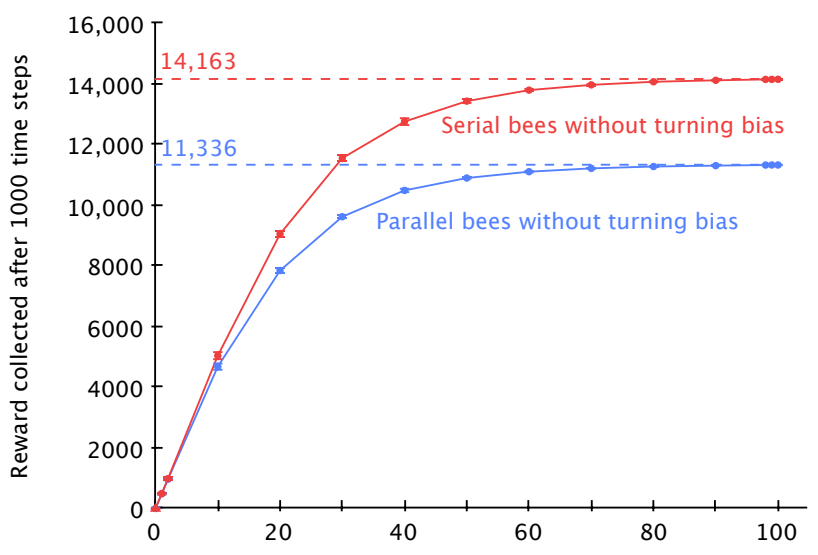

Percentage of space occupied by targets (fixed $0 \%$ distractors)

Figure 5: Graph of nectar rewards collected by colonies of abees versus the percentage of grid cells occupied by target flowers. Data points are the mean of 20 simulations; error bars \pm 1 std. dev.

\section{Target and distractor experiments}

In these experiments $10 \%$ of the foraging patch was randomly filled with targets. Distractor density was increased systematically from $0 \%$ to $90 \%$ of the total number of grid squares. The distractors were randomly distributed among the cells unoccupied by targets. The effects of this increase on the amount of nectar collected by colonies of parallel and serial abees executing ordinary random walks and biased random walks are shown (Fig. 6).

Considering first the a-bees executing an ordinary random walk. For distractor densities less than $1 \%$, the serial scan mechanism outperforms the parallel scan (see Fig. 5 above, since the Target only experiment at $0 \%$ distractors, matches the experiment here at $0 \%$ distractors). However, parallel scan takes over as the most efficient mechanism at higher distractor densities, even where the number of distractors is much less than the number of targets. Parallel scan is clearly more efficient than the serial scan as distractor flower density increases beyond target density.

The trends for a-bees conducting a biased random walk correspond directly to those for a-bees conducting an ordinary random walk, but with greater overall success for the former over the latter. The directional bias appears to enhance the speed with which a-bees' locate unvisited targets. It does this in equal proportion for a-bees using parallel or serial visual scan and didn't change the relative success of these mechanisms.

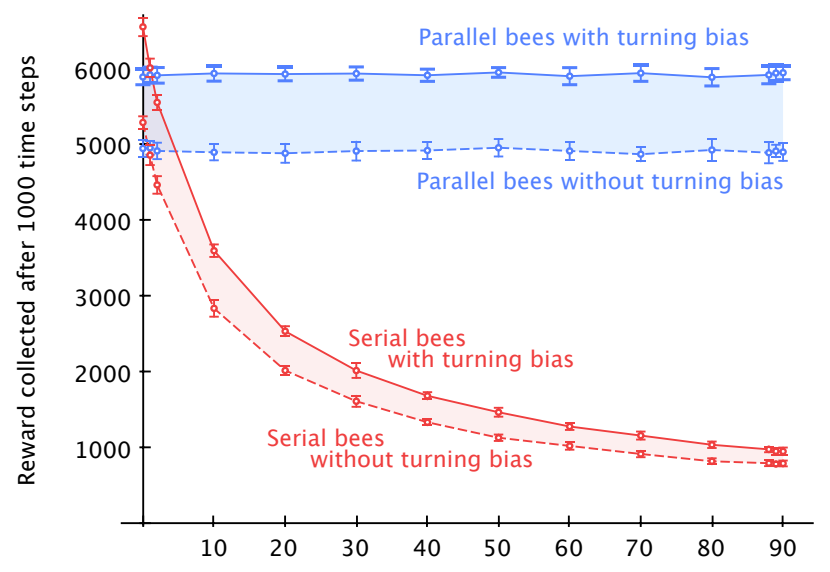

Percentage of space occupied by distractors (fixed 10\% targets)

Figure 6: Graph of nectar rewards collected by colonies of abees versus the percentage of grid cells occupied by distractor flowers. Target flower density was fixed at $10 \%$. Data points are the mean of 20 simulations; error bars \pm 1 std. dev.

\section{Discussion, Conclusions and Future Work}

In the current study we tested for the relative success of a serial scan as occurs in honeybees, and a parallel scan typical of bumblebees, considering previous evidence that honeybees evolved to forage in more tropical environments and bumblebees in temperate environments (Clark 1994; Dornhaus and Chittka 2004; Heinrich 2004; Dyer, Spaethe et al. 2008). This lead us to hypothesise that honeybees' serial scan may be more effective in environments where targets were not interspersed among distractors, and that bumblebees' parallel scan would be relatively more effective for foraging in heterogeneous environments where distractors and targets were intermingled. These hypotheses were supported by our simulation results as follows.

\section{Target only experiments}

The ratio of nectar collected by the parallel a-bees to that collected by the serial a-bees in an environment without distractors approaches 11,336:14,163 $=4: 5$. The serial mechanism is increasingly superior as target density increases. 
This ratio can be derived analytically in the absence of distractors and a neighbourhood increasingly saturated with targets. For each traversal of the procedure in Fig. 2 a parallel a-bee requires ParallelTime + MoveTime + VisitTime to collect a reward unit. With our parameters (Tab. 1) this amounts to $3+1+1=5$ simulation time steps. A serial a-bee's traversal of the procedure in Fig. 3 requires SerialTime + MoveTime + VisitTime $=2+1+1=4$ simulation time steps to collect a reward unit. Hence, a serial a-bee takes $4 / 5^{\text {ths }}$ the time of a parallel a-bee to collect a reward unit in this scenario.

We could have set our simulation parameters so that the abees' decision time wasn't the most time-consuming activity in its foraging cycle. This would potentially be more biologically plausible given that decision times as measured by Morawetz and Spaethe (2012) were generally less than 1.5 seconds (bumblebees) and 1.2 seconds (honeybees), and that the sum of travel and flower handling times may exceed these values in the real world by many seconds (Chittka, Gumbert et al. 1997; Chittka 2002). However, these time handling factors may also be complicated by interactions with other foraging insects in complex and competitive environments where being faster may allow the collection of rewards before competitors diminish resources (Burns and Dyer 2008). The impact of travel and flower handling times for real honeybees and bumblebees may impact on their relative success in different environments since each species may conceivably have differences in flight speed and flower handling skill under various real world conditions. But the scope of our experiments was only to test differences between the scanning mechanisms these species have been shown to employ. Hence, we neutralise flower handling and travel time differences by assigning equal values to VisitTime and MoveTime parameters for the two a-bee species.

It was found that real bumblebees reduce their decision time slightly, by a statistically significant amount, as the number of visible targets increases from one to four (Morawetz and Spaethe 2012). Hence, the gap we report between parallel and serial mechanisms may be decreased slightly from that shown for our a-bees as target density increases (Fig. 5).

\section{Target and distractor experiments}

A distractor density of only $1 \%$ is sufficient to level the effectiveness of the serial and parallel scanning mechanisms. Any increase beyond this improves the parallel scanning mechanism's superiority over the serial scan. This is interesting as it implies that in many biologically plausible scenarios, parallel search would be the more efficient approach for collecting nutrition from rewarding flowers whilst avoiding dissimilar colored distractors. Why then mightn't honeybees have evolved a more efficient visual scan? A potential answer is provided in figure 5 where, in an environment with no distractors (as would be present in many tropical scenarios where large trees flower) fast serial search is more effective. Indeed, in studies that have compared the efficiency of honeybees at collecting nutrition in temperate and tropical environments, and where the capacity of the honeybees to recruit nest mates to share in resource gathering was experimentally manipulated, research has found honeybees to be most effective in tropical environments (Dornhaus and Chittka 2004). Thus our current findings that there are biologically plausible conditions that best suit either a parallel or serial search mechanism, adds weight to the possibility of environmental conditions leading to the evolution of different visual capabilities in different bee species (Dyer, Spaethe et al. 2008; Morawetz and Spaethe 2012).

As noted above, the biased random walk has no impact on the relative success of the scanning mechanisms. However in absolute terms, in our simulations, the turning bias we implemented improved the parallel a-bees' foraging success by $20.48 \%$ (std. dev. 0.85 ) and the serial a-bees' success by $24.7 \%$ (std. dev. 2.4). This certainly leaves it open for those interested in optimal foraging to simulate more biologically plausible navigation schemes, however this was outside the scope of our study.

\section{Future work}

Our findings suggest it would be valuable to compare tropical and temperate floral distributions and how these may have affected the processing capabilities of different bee species. This work is potentially useful because it has been suggested that climate change may lead to either spatial or temporal mismatches in the availability of flower resources and pollinators (Hegland, Nielsen et al. 2009), and indeed such mismatches could be influenced by the visual capacities of bees. Currently this is unknown, and testing bee species of high pollination value with flowers of different patchiness may provide important insights for future resource management.

\section{Acknowledgements}

This research was supported under Australian Research Council's Discovery Projects funding scheme (project numbers DP0878968, DP0987989, DP130100015).

\section{References}

Axelrod, R. (2003). "Advancing the art of simulation in the social sciences." Japanese Journal for Management Information Systems - Special Issue on Agent-Based Modeling: 19.

Burns, J. G. (2005). "Impulsive bees forage better: the advantage of quick, sometimes inaccurate foraging decisions." Animal Behaviour 70: e1-e5.

Burns, J. G. and A. G. Dyer (2008). "Diversity of speed accuracy strategies benefits social insects." Current Biology 18: R953R954.

Chittka, L. (2002). "The influence of intermittent rewards on learning to handle flowers in bumblebees." Entomologia generalis 26(2): 85-91.

Chittka, L., A. Gumbert and J. Kunze (1997). "Foraging dynamics of bumble bees: correlates of movements within and between plant species." Behavioural Ecology 8: 239-249. 
Chittka, L., J. D. Thomson and N. M. Waser (1999). "Flower constancy, insect psychology, and plant evolution." Naturwiss 86: 361-377.

Clark, D. A. (1994). Plant demography. in La Selva-ecology and natural history of a neotropical rain forest. L. A. McDade, K. S. Bawa, H. A. Hespenheide and G. S. Hartshorn. Chicago, University of Chicago Press: 90-105.

Dafni, A. (1984). "Mimicry and deception in pollination." Annual Review of Ecology and Systematics 15: 259-278.

DeAngelis, D. L. and W. M. Mooij (2005). "Individual-based modelling of ecological and evolutionary processes." Annu. Rev. Ecol. Evol. Syst. 36: 147-168.

Dorin, A., K. B. Korb and V. Grimm (2008). Artificial-Life Ecosystems: What are they and what could they become? Eleventh International Conference on Artificial Life. S. Bullock, J. Noble, R. A. Watson and M. A. Bedau, MIT Press: 173-180.

Dornhaus, A. and L. Chittka (1999). "Evolutionary origins of bee dances." Nature 401: 38.

Dornhaus, A. and L. Chittka (2004). "Why do honeybees dance?" Behavioural Ecology and Sociobiology 55: 395-401.

Dornhaus, A., F. Klügl, C. Oechslein, F. Puppe and L. Chittka (2006). "Benefits of recruitment in honey bees: effects of ecology and colony size in an individual-based model." Behavioural Ecology 17(336-344).

Duchateau, M. J. and H. H. W. Velthuis (1988). "Development and reproductive strategies in Bombus terrestris colonies." Behaviour 107: 186-207.

Dyer, A. G., S. Boyd-Gerny, S. McLoughlin, M. G. P. Rosa, V. Simonov and B. B. M. Wong (2012). "Parallel evolution of angiosperm colour signals: common evolutionary pressures linked to hymenopteran vision." Proceedings of the Royal Society of London B 279: 3605-3615.

Dyer, A. G., A. Dorin, V. Reinhardt and M. G. P. Rosa (2012). "Colour reverse learning and animal personalities: the advantage of behavioural diversity assessed with agent-based simulations." Nature Precedings(March): 20.

Dyer, A. G., A. C. Paulk and D. H. Reser (2011). "Colour processing in complex environments: insights from the visual system of bees." Proceedings of the Royal Society B 278:952-959. Dyer, A. G., J. Spaethe and S. Prack (2008). "Comparative psychophysics of bumblebee and honeybee colour discrimination and object detection." Journal of Computational Physiology A 194: 614-627.

Frisch, K. v. (1967). "The Dance Language and Orientation of Bees". Cambridge, USA, Harvard University Press.

Giurfa, M. (2004). "Conditioning procedure and color discrimination in the honeybee Apis mellifera." Naturwissenschaften 91: 228-231.

Giurfa, M., J. Núñez and W. Backhaus (1994). "Odour and colour information in the foraging choice behaviour of the honeybee." Journal of Comparative Physiology A 175(6): 773-779.

Grimm, V. (1999). "Ten years of individual-based modelling in ecology: what have we learned and what could we learn in the future?" Ecological Modelling 115: 129-148.
Grimm, V. and S. F. Railsback (2005). "Individual-based Modeling and Ecology", Princeton University Press.

Grimm, V., E. Revilla, U. Berger, F. Jeltsch, W. M. Mooij, S. F. Railsback, H.-H. Thulke, J. Weiner, T. Wiegand and D. L. DeAngelis (2005). "Pattern-Oriented Modelling of Agent-Based Complex Systems: Lessons from Ecology." Science 310: $987-$ 991.

Hegland, S. J., A. Nielsen, A. Lázaro, A. L. Bjerknes and Ø. Totland (2009). "How does climate warming affect plantpollinator interactions?" Ecological Letters 12: 184-195.

Heinrich, B. (2004). "Bumblebee economics". Cambridge, Harvard University Press.

Hogeweg, P. and B. Hesper (1983). "The Ontogeny of the Interaction Structure in Bumble Bee Colonies: A MIRROR Model." Behavioural Ecology and Sociobiology 12: 271-283.

Huston, M., D. DeAngelis and W. Post (1988). "New Computer Models Unify Ecological Theory." BioScience 38(10): 682-691.

Judson, O. P. (1994). "The rise of the individual-based model in ecology." Trends in Ecology and Evolution 9(1): 9-14.

Kjøhl, M., A. Nielsen and N. C. Stenseth (2011). "Potential Effects of Climate Change on Crop Pollination". Rome, Food and Agricultural Organization.

Lythgoe, J. N. (1979). "The ecology and vision". Oxford, U.K., Clarendon Press.

Morawetz, L. and J. Spaethe (2012). "Visual attention in a complex search task differs between honeybees and bumblebees." Journal of Experimental Biology 215: 2515-2523.

Pyke, G. H. (1984). "Optimal Foraging Theory: A Critical Review." Annual Review of Ecology and Systematics 15:523-575.

Srinivasan, M. V. (2011). "Honeybees as a model for the study of visually guided flight, navigation, and biologically inspired robotics." Physiological Reviews 91(2): 413-460.

Streinzer, M., H. F. Paulus and J. Spaethe (2009). "Floral colour signal increases short-range detectability of a sexually deceptive orchid to its bee pollinator." Journal of Experimental Biology 212: $1365-1370$.

Treisman, A. M. and G. Gelade (1980). "A feature-integration theory of attention." Cognitive Psychology 12: 97-136.

Treisman, A. M. and S. Gormican (1988). "Feature analysis in early vision: evidence from search asymmetries." Psychological Review 95: 15-48.

VanRullen, R., T. Carlson and P. Cavanagh (2007). "The blinking spotlight of attention." Proceedings of National Academy of Science USA 104: 19204-19209.

Vries, H. d. and J. C. Biesmeijer (1998). "Modelling collective foraging by means of individual behaviour rules in honey-bees." Behavioural Ecology and Sociobiology 44(2): 109-124.

Waddington, K. D. (1980). "Flight Patterns of Foraging Bees Relative to Density of Artificial Flowers and Distribution of Nectar." Oecologia 44(2): 199-204.

Wertlen, A. M., C. Niggebrugge, M. Vorobyev and N. H. d. Ibarra (2008). "Detection of patches of coloured discs by bees." Journal of Experimental Biology 211: 2101-2104. 